\title{
El ejercicio físico como un todo para la salud humana
}

Physical exercise as a whole for human health.

Efraín Velasteguí López. ${ }^{1}$ Lic. Joel García Espinosa. ${ }^{2}$ Est. Laidee Mercedes Espinosa Martínez. ${ }^{3}$ Est. Walter Manuel Martínez Capdevila. ${ }^{4}$ Est. Yenny Hernández. ${ }^{5}$ \& Est. Yaniuska Amalia González Suarez. ${ }^{6}$

\begin{abstract}
.
Physical exercise constitutes for the organism a change in the conditions of equilibrium of the internal environment, it is given by the physical capacities which constitute basic qualities in the optimal physical condition of each individual, being the training the factor that offers the possibility of improving the physical capacities which constitute foundations for learning and perfecting the
\end{abstract}

\section{Resumen.}

El ejercicio físico constituye para el organismo un cambio en las condiciones de equilibrio del medio interno, está dado por las capacidades físicas las que constituyen cualidades básicas en la óptima condición física de cada individuo, siendo el entrenamiento el factor que ofrece la posibilidad de mejorar las capacidades físicas las cuales constituyen fundamentos para el

1 Ciencia digital, Ambato, Ecuador, luisefrainvelastegui@cienciadigital.org https://orcid.org/0000-0002-7351-0108

${ }^{2}$ Ciencias Médicas Mayabeque, Mayabeque, Cuba, jgarcia@infomed.sld.cu iD https://orcid.org/0000-00027351-0109

${ }^{3}$ Ciencias Médicas Mayabeque, Mayabeque, Cuba, lmercedes@infomed.sld.cu 0002-7351-0110

${ }^{4}$ Ciencias Médicas Mayabeque, Mayabeque, Cuba, wmartinez@infomed.sld.cu 0002-7351-0111

${ }^{5}$ Ciencias Médicas Mayabeque, Mayabeque, Cuba, yhernandez@infomed.sld.cu 0002-7351-0112

${ }^{6}$ Ciencias Médicas Mayabeque, Mayabeque, Cuba, ygonzalez@infomed.sld.cu 0002-7351-0113

https://orcid.org/0000-

https://orcid.org/0000-

https://orcid.org/0000-

https://orcid.org/0000- 
motor actions for life. A quality physical exercise should be developed based on the morpho-functional conditions that the organism possesses. The adaptations of the organism to the change will suppose a physical-biological benefit for the individual as long as the exercise is carried out in adequate conditions. Physical exercise has perfected men's ability to move. Direct contact with physical exercise determines that special states and feelings are experienced with regard to the activity of the whole organism and particularly with muscular activity. By practicing it systematically, the person adopts a behavior that adapts to the norms of sports activity. The physical exercise is in close relationship with the abilities that are (strength, endurance, speed and flexibility) and the warm-up which is made up of joint mobility exercises, activities to raise the pulse and stretching exercises and movements related to activity.

Keywords: Physical exercise, physical abilities, warm up aprendizaje y el perfeccionamiento de las acciones motrices para la vida. Un ejercicio físico de calidad debe desarrollarse sobre la base de las condiciones morfo-funcionales que posea el organismo. Las adaptaciones del organismo al cambio van a suponer un beneficio físico-biológico para el individuo siempre y cuando el ejercicio se realice en condiciones adecuadas. El ejercicio físico ha perfeccionado la capacidad de movimientos de los hombres. El contacto directo con el ejercicio físico, determina que se experimenten estados y sentimientos especiales con respecto a la actividad de todo el organismo y particularmente con la actividad muscular. Al practicarlo sistemáticamente la persona adopta un comportamiento que se adapta a las normas de la actividad deportiva. El ejercicio físico está en estrecha relación con las capacidades que son (fuerza, resistencia, rapidez y flexibilidad) y el calentamiento el cual se compone por los ejercicios de movilidad articulas, las actividades para elevar el pulso y los ejercicios de estiramiento y movimientos relativos a la actividad.

Palabras claves: Ejercicio físico, capacidades físicas, calentamiento.

\section{Introducción.}

La actividad física estuvo asociada a la salud de las personas desde tiempos remotos. Los deportistas de la antigua civilización griega, por ejemplo, ocupaban un lugar destacado dentro de la sociedad y se llegaron a endiosar sus hazañas atléticas. A lo largo de la historia se fue relacionando la longevidad de ciertas poblaciones del planeta con estilos de vida activos y adecuada alimentación. El ejercicio físico constituye para el organismo un cambio en las condiciones de equilibrio del medio interno. Las adaptaciones del organismo al cambio van a suponer un beneficio físico-biológico para el individuo siempre y cuando el ejercicio se realice en condiciones adecuadas. La actividad física estuvo asociada a la salud de las 
personas desde tiempos remoto. Los deportistas de la antigua Civilización Griega, ocupaban un lugar destacado dentro de la sociedad y se llegaron a endiosar sus hazañas atléticas. A lo largo de la historia se fue relacionando la longevidad de ciertas poblaciones del planeta con estilos de vida y adecuada alimentación.

El ejercicio físico es la actividad recreativa, que se realiza en momentos de tiempo libre, es decir, fuera del trabajo o actividad laboral. El ejercicio físico implica la realización de movimientos corporales planificados y diseñados específicamente para estar en forma física y gozar de buena salud. El termino de ejercicio físico es el de gimnasia, baile, deporte y educación física.

\section{Desarrollo}

El ejercicio físico puede ayudar no sólo a conseguir y mantener un estado de completo bienestar físico, mental y social, sino también a disfrutar plenamente de la vida. Por ello se realizó un estudio con el objetivo de valorar la efectividad del mismo en la longevidad.

El principio del ejercicio físico se basa en el hecho demostrado de que hacer un ejercicio provoca una serie de cambios fisiológicos en el organismo que son responsables de la mejora de nuestro estado de salud. Un programa de ejercicio físico realizado regularmente debe de ser un estímulo para conseguir una adaptación y mejora progresiva de estos aspectos fisiológicos.

Principio de sobrecarga: Nos declara que hace falta hacer un ejercicio con cargas(intensidades) superiores a las que nuestros sistemas fisiológicos están acostumbrados a soportar. La explicación es simple: En nuestra vida cotidiana realizamos constantemente ciertos trabajos a los que nuestro cuerpo está plenamente acostumbrado. Si al realizar la sección de ejercicios no vamos un poco más allá del esfuerzo que estas nos suponen, será muy difícil que podamos obtener ningún ejercicio de la práctica.

Principio de especificidad: Nos propone que las respuestas fisiológicas, metabólicas y de adaptación al programa de ejercicio son especificas al tipo de los grupos musculares vinculados. Es evidente que según el ejercicio o deporte que practiquemos trabajaremos más unas artes del cuerpo que otras, así como si realizamos un ejercicio intenso y corto no obtendremos resultados para la práctica de ejercicios menos intensos, pero de mayor duración. A Nivel de hacer ejercicios para potenciar nuestro estado de salud, la premisa será la de hacer prácticas deportivas que involucran el mayor número de grupos musculares (brazos, piernas, abdomen, espalda...), haciendo un trabajo de mediana intensidad y media duración (ritmo que podamos mantener al menos durante treinta minutos).

Principio de progresión: Nos dice que la carga(intensidad) del ejercicio debe aumentar progresivamente durante el trascurso de un programa de ejercicio, con fines de obtener 
mejoras. Es decir, que a medidas que vayamos haciendo sesiones, la intensidad del ejercicio deberá ir incrementándose. Esto no quiere decir que cada día que vayamos a hacer ejercicios debamos sufrir más, o que debamos correr cada día 5minutos más que el día anterior, ya que el cuerpo se va adaptando a este aumento de intensidad, soportando cada día un mayor esfuerzo y la progresión debe ser paulatina, pudiendo hacer unas cuantas sesiones con intensidad similar antes de subir las cargas de trabajo.

Principio de valores iniciales: Los individuos con un nivel de capacidad bajo (es decir, poco acostumbrado a hacer ejercicio) mejoran más y de forma más rápida que los individuos con un nivel de capacidad alto (o que ya están acostumbrados a realizar ejercicios) Con este enunciado no estamos diciendo nada más que como más ejercicio realicemos, mayor es el grado de adaptaciones de nuestro cuerpo, y a mayor grado de adaptación menos margen de mejores tenemos .Esto, pero, no significa que después de un tiempo seguimiento un programa de ejercicio no obtengamos mejoras. Si lo hacemos correctamente siempre las obtendremos, aunque en menor grado a medida que pasa el tiempo.

Principios de variabilidad individual: Cada persona responde de forma diferente a un programa de ejercicio en función de factores como la edad, el nivel de capacidad inicial o su estado de salud. El ejercicio físico s como la dieta: debe de ser pautado y confeccionado individualmente para cada persona, ya que cada uno tendrá sus condicionantes personales que determinaran como debe de ser el programa a seguir. Al igual que la dieta, existe la figura de un profesional cualificado y especializado en este trabajo, es el licenciado o graduado en Ciencias de la Actividad y Deporte.

Las enfermedades del corazón y las arterias ocupan un triste primer lugar como causa de mortalidad en el mundo occidental. En su aparición y desarrollo están involucrados múltiples factores de riesgo dentro de los cuales el tabaquismo, las alteraciones del colesterol, la hipertensión arterial, el sedentarismo, la diabetes, el estrés mental y la obesidad ocupan un lugar de relevancia. El ejercicio físico regular contribuye a combatir todos estos factores y más aún, al actuar directamente sobre el corazón y los vasos sanguíneos, es uno de los elementos más eficaces para prevenir la aparición y la progresión de la enfermedad. En las personas que ya han sufrido una enfermedad arritmia cardiaca, se ha comprobado que el ejercicio colabora en el tratamiento, mejorando la calidad y la cantidad de años vivos.

El ejercicio físico presenta beneficios para mejorar la calidad de vida de las personas que son beneficios generales y efecto con beneficios particulares, por ejemplo:

\section{Beneficios Generales:}

- Aumenta la resistencia a la fatiga e incrementa la capacidad para el trabajo físico y mental. 
- Ayuda a combatir la ansiedad, la depresión y el estrés mental.

- Mejora la capacidad para conciliar el sueño.

- Provee una manera sencilla para compartir actividades con amigos y familiares contribuyendo a mejorar aspectos sociales.

- Ofrece mayor energía para las actividades diarias.

- Tonifica los músculos e incrementa su fuerza.

- Mejora el funcionamiento de las articulaciones

- Contribuye a la pérdida de peso cuando esto es necesario.

\section{Efecto y Beneficios Particulares:}

\section{En el Corazón:}

- Disminuye la frecuencia cardiaca de reposo y, cuando se hace un esfuerzo, aumenta la cantidad de sangre que expulsa el corazón en cada latido. De esta manera la eficiencia cardiaca es mayor gastando menos energía para trabajar.

- Estimula la circulación dentro del musculo cardiaco favoreciendo la alimentación del corazón.

\section{El Sistema Circulatorio:}

- Contribuye a la reducción de la presión arterial.

- Aumenta la circulación en todos los músculos.

- Disminuye la formación de coágulos dentro de las arterias con lo que previene la aparición de infartos y de trombosis cerebrales.

- Actúa sobre el endotelio, que es la capa de células que tapiza por dentro a las arterias segregando sustancias de suma importancia para su correcto funcionamiento, mejorando su actividad y manteniéndolo sano y vigoroso. Todo esto por una acción a través de hormonas que se liberan con el ejercicio.

- Mejora el funcionamiento venoso previniendo la aparición de varices.

\section{El Metabolismo:}

- Aumenta la capacidad de aprovechamiento de oxigeno que la llega al organismo por la circulación 
- Aumenta la actividad de las enzimas musculares, elementos que permiten un mejor metabolismo del musculo y por ende una menor necesidad de exigencia de trabajo cardiaco.

- Aumenta el consumo de grasa durante la actividad con lo que contribuye a la pérdida de peso.

- Colabora a la disminución del colesterol total y del colesterol LDL(malo) con aumento del colesterol HDL(bueno).

- Mejora la tolerancia a la glucosa favoreciendo el tratamiento de la diabetes.

- Incrementa la secreción y trabajo de diferentes hormonas que contribuyen a la mejoría de las funciones del organismo.

- Colabora en el mantenimiento de una vida sexual plena.

- Mejora la respuesta inmunológica ante infecciones o agresiones de distinto tipo.

\section{En el tabaquismo:}

- Los individuos que realizan el ejercicio físico dejan el hábito de fumar con mayor facilidad y hay una relación inversa entre ejercicio físico y tabaquismo.

\section{Aspectos Psicológicos:}

- Aumenta la sensación de bienestar y disminuye el estrés mental. Se produce liberación de endorfinas, sustancias del propio organismo con estructuras químicas similar a morfinas, que favorecen el sentirse bien después del ejercicio (sin por supuesto, los efectos negativos de la droga).

- Disminuye el grado de agresividad, ira, ansiedad, angustia y depresión.

- Disminuye la sensación de fatiga.

\section{El aparato locomotor:}

- Aumenta la elasticidad muscular y articular.

- Incrementa la fuerza y la resistencia de los músculos.

- Previene la producción de osteoporosis.

- Previene el deterioro muscular producido por los años.

- Facilita los movimientos de la vida diaria.

- Contribuye a la mejor independencia de las personas mayores. 
Entre los riesgos del ejercicio físico esta la sobrecarga al aparato cardiovascular, donde el ejercicio físico puede desenmascarar enfermedades latentes y generar algún problema cardiaco. Sin embargo, los beneficios del ejercicio con regularidad superan ampliamente a los eventuales riesgos. Más aun, los pocos frecuentes eventos cardiacos se producen en su mayoría ante ejercicios vigorosos, para los cuales, por otra parte, es necesario tener un examen cardiovascular previo.

También es posible la aparición de lesiones traumatológicas generadas por el ejercicio vigoroso. Existen métodos y conductas para minimizar los riesgos. Los riesgos más frecuentes de la actividad física y los deportes están relacionados con las lesiones musculares, óseas y articulares. Estos eventos ocurren, por lo general, cuando el ejercicio es demasiado intenso para esa persona o bien cuando se comienza un deporte desde de mucho tiempo de inactividad.

Es necesario tomar sientas precauciones destinadas a la prevención de este tipo de lesiones. Uno de los puntos más importantes se relaciona con el periodo de calendario previo al inicio de la actividad y 1 vuelta al frio antes de suspenderla.

También es de suma importancia no imponerse metas demasiado exigentes para los primeros tiempos de la actividad. Esto lo llevaría a realizar ejercicios bruscos y muy intensos que pueden producir lesiones.

No es conveniente continuar con el ejercicio si existen dolores musculares o en las articulaciones. Lo mejor es suspender la actividad por lo menos hasta que un médico aconseje al respecto.

El ejercicio físico aborda varios consejos para los días calurosos y días fríos entre ellos están:

\section{En días calurosos:}

- Trate de efectuar los ejercicios temprano en la mañana o a últimas horas de la tarde.

- Acostúmbrese a beber líquidos antes, durante y después de la actividad.

- Use ropa liviana y suelta tratando de tener la mayor parte del cuerpo descubierta.

- Tenga en cuenta síntomas precoces de Golpe de Calor como los mareos, debilidad, cansancio exagerado o dolor de cabeza. En este caso debe suspender la actividad tomar líquidos y permanecer en lugares frescos y aireados. La enfermedad por calor puede llegar a ser muy grave.

\section{En días fríos:}


- Utilice varias prendas para abrigarse. De esta manera le será más útil quitarse alguna cuando la temperatura de su cuerpo comience a aumentar.

- Utilice guantes para proteger las manos y gorras y bufandas para evitar perdida de calor por la cabeza y el cuello.

Independientemente de las influencias de las propiedades orgánicas individuales, existen tres factores que determinan la rapidez, facilidad y magnitud con que pueden desarrollarse las capacidades físicas:

- La particularidad de las influencias externas dirigidas al desarrollo de esas capacidades motoras.

- Las particularidades desde el punto de vista ontogenético que tiene cada individuo.

- La particularidad de que una misma actividad puede desarrollar diferentes capacidades físicas.

Estas capacidades físicas se pueden dividir en dos grupos:

1. Capacidades condicionales y flexibilidad.

2. Capacidades coordinativas.

\section{Capacidades condicionales o flexibilidad:}

Fuerza: Es la habilidad de moverse, y permite la capacidad para producir la máxima tensión muscular que los músculos desarrollan durante el trabajo. También es la capacidad para vencer una existencia por medio de un esfuerzo muscular.

En todos los deportes se utilizan un sinfín de técnicas encaminadas a conseguir un incremento en la capacidad de contracción muscular del ejecutante, lo que corresponde al entrenamiento de la fuerza. La naturaleza de un ejercicio de potencia está encaminada a desarrollar algunos de los tipos de fuerza que se presentan en la contracción muscular, y que según Fleishman son:

Fuerza Explosiva: Responsable de la ejecución de los movimientos muy rápidos, y de arranque explosivo. La práctica de la totalidad de las técnicas de Karate, sobre todo aplicadas a la competición, se incluyen en la fuerza explosiva. En centésimas de segundo, el cerebro debe decidir sobre la acción que se va a realizar y manda la información a los músculos para que actúen contrayéndose y provocando el movimiento

Fuerza Dinámica: Es la capacidad de mantener una posición en contracción muscular, o también de realizar repeticiones de ejercicios del mismo tipo (carreras, flexiones, 
técnicas de puño y de abrazo) Esta cualidad debe ser progresivamente entrenada por el karate para contribuir al desarrollo de la fuerza mantenida

Fuerza Estática: En ella se valora la capacidad para ejercer una fuerza determinada sin que haya un acortamiento muscular, y, por tanto, sin que haya movimiento del segmento corporal que trabajamos. Otros autores denominan a esta propiedad como fuerza pura, pues en ella el musculo adquiere una tensión máxima para intentar vencer una resistencia demasiado elevada para él, y que no va a poder mover. La medida de esta cualidad se realiza en los dinamómetros.

Rapidez: Es una cualidad física determinante para el rendimiento deportivo. Estando presente de alguna forma en todas las manifestaciones del deporte, saltar, correr, levantar. Además, también podemos decir que es la capacidad con que se mueve un cuerpo con mayor velocidad al realizar un movimiento u otra actividad.

La rapidez es un factor determinante en los deportes explosivos, por ejemplo, saltos y la mayoría de los deportes de campo, mientras que en las competiciones de resistencia su función como factor determinante parece reducirse. Entre los tipos de esta están:

La rapidez de reacción: Es aquella capacidad de reaccionar muy rápidamente ante un movimiento (por ejemplo, en la esgrima).

La rapidez de traslación: Consiste en la capacidad de moverse con la mayor rapidez posible.

La resistencia de la rapidez: Es la capacidad de no permitir el descenso de la rapidez de traslación en una distancia corta, o de poder realizar, un y otra duración (por ejemplo, en los distintos tipos de juegos con pelota).

El entrenamiento de la rapidez abarca tres grupos de ejercicios dentro de los cuales están:

- El ejercicio de reacción, para el mejoramiento de la reacción nerviosa y muscular (por ejemplo, ante la señal de arrancado o ante un contrario).

- El ejercicio de aceleración, para el mejoramiento de esta capacidad de aceleración.

- El ejercicio de carrera rápida, para el mejoramiento de la rapidez de la carrera. Orientación para los ejercicios de carrera.

Resistencia: Es la cualidad que nos permite soportar y aguardar un esfuerzo durante el mayor tiempo posible. El desarrollo natural (sin entrenamiento) de la resistencia se produce en diferentes etapas: 
- De los 8 a 12 año hay un crecimiento mantenido de la capacidad de los esfuerzos moderados y continuos.

- Desde los 18 años a los 22 se alcanza el límite máximo de la resistencia.

- Ya partir de los 30 años va decreciendo

Entre los tipos de resistencias están:

Resistencia Aeróbica: Es un esfuerzo de resistencia cuando el oxígeno que puede llegar a los músculos que trabajan es suficiente para realizar el ejercicio. Un ejemplo puede ser ir en bicicleta y, como podemos intuir, debería ser un esfuerzo de intensidad moderada.

Resistencia Anaeróbica: Supongamos que empezamos a pedalear más rápido. Nuestros músculos necesitaran más oxígeno y por tanto empezaremos a enviar más sangre. Si el ejercicio es muy intenso, el oxígeno que llega es insuficiente y vamos cayendo progresivamente en el agotamiento. Estos ejercicios físicos, realizados con déficit o incluso una falta total de oxígeno, se les llama de resistencia anaerobia. Son esfuerzos muy intensos y que, debido a la falta de oxígeno, tienen corta duración. Para controlar nuestro esfuerzo tomaremos las pulsaciones.

La resistencia anaeróbica se diferencia en resistencia anaeróbica

- Aláctica: Los esfuerzos son intensos y de muy corta duración (0-16 s). La presencia de oxígeno es prácticamente nula. La utilización de sustrato energético (ATP, PC) no producen sustancia de desecho.

- Láctica: Esfuerzos y de corta duración (15s-2min), la utilización de sustrato energético produce sustancias de desecho (ácido láctico) que se va acumulando y causa de forma rápida la fatiga. La adaptación más importante que se produce en el organismo con el entrenamiento de la resistencia es la mejora del sistema cardiorrespiratorio. El trabajo de resistencia produce adaptaciones funcionales en el corazón. El entrenamiento de la resistencia aeróbica agrada las paredes y cavidades internas del corazón, mientras que el entrenamiento de la resistencia anaeróbica provoca el engrosamiento de la pared del muslo cardiaco. De esta forma, cuanto mayor y más grande sea el corazón, más cantidad de sangre podrá bombear en cada pulsación.

Flexibilidad: A diferencia de las otras cualidades físicas, la flexibilidad es una capacidad que se va perdiendo desde que se nace.

En esta cualidad las mujeres poseen mayores niveles de flexibilidad que los hombres. Sus articulaciones son más laxas y permiten mayor movimiento, además poseen menos tono muscular que contribuyen aún más. 
La flexibilidad es necesaria en muchos deportes en los cuales se requiere amplios movimientos articulares como el Taekwondo, pero también se necesita en deportes en los que se debe desarrollar fuerza explosiva, pues cuanta más flexibilidad mayor será el recorrido y por tanto mayor el impulso que se obtendrá.

Es importante para todos los deportistas entrenar la flexibilidad, porque aparte de las razones comentadas, poseer flexibilidad previene de muchas lesiones. La flexibilidad se entrena por medios de los llamados estiramientos, que muchas veces se incluyen en los ejercicios de calentamiento previos al inicio de la competición o al entrenamiento.

Las partes del cuerpo que intervienen en la ejecución de una acción se mueve en el punto apropiado, en el momento oportuno y en la secuencia correcta.

La destreza era conocida como la capacidad para resolver rápida y adecuadamente las tareas motoras. Así, por ejemplo, un esquiador que supera dificultades elevados, es considerado una persona discreta. El jugador de(Hand-ball) que se desenvuelve exitosamente por medio de fintas, saltos o tiros con caídas, o un boxeador que contrarresta el ataque del adversario, también posee un grado de destreza. Cada disciplina deportiva para el buen curso del movimiento exige del atleta una buena flexibilidad. Un desarrollo multilateral implica el desarrollo de la flexibilidad de todas las articulaciones en todas las direcciones. Naturalmente el entrenamiento de la flexibilidad ha de ser aún más profundo(amplio) en aquellas disciplinas deportivas, para las cuales la misma tenga una mayor significación.

Es importante saber la significación de la flexibilidad que tiene para el aumento del rendimiento en tu disciplina deportiva, además de conocer cómo trascurre los movimientos y en qué parte del movimiento es especialmente importante la flexibilidad. De gran significación es saber en qué articulación y en qué dirección se debe mejorar la amplitud del movimiento.

En el entrenamiento correcto de los ejercicios se debe prestar atención a lo que se conoce sobre el entrenamiento de la fuerza. Se debe repetir varias veces los ejercicios de flexibilidad. Un ejercicio realizado por una sola vez no ayuda. ¡Por lo tanto se entrena en series! Por lo que es recomendable seguir las reglas que son las siguientes:

- Entrenar lo más menudo posible. La flexibilidad desaparee cuando se entrena con mucha irregularidad (con mucha diferencia de tiempo)

- Realizar los ejercicios de flexibilidad de forma tal que mediante repetidas oscilaciones se logre progresivamente la amplitud máxima del movimiento. No obstante, puede entrenarse también de manera que, por ejemplo, durante la flexión del tronco se permanezca en la posición de flexión hasta contar hasta tres. Solo entonces se retorna a la posición inicial. 
- No realizar solo aquellos ejercicios que mejoran con la influencia del peso corporal (ejemplo en esparranca) o con la ayuda del ejercicio que fortalecen los músculos que intervienen en la realización del movimiento.

Las Capacidades Coordinativas son las que están relacionadas con la capacidad de conducción, control y regulación del movimiento, es decir con los procesos informacionales. Estas capacidades forman parte de las capacidades físicas, al desarrollar hay que incluirlas dentro de la preparación física general y especial, que junto a la preparación técnico-táctica y psicológica del atleta son las encaminadas a desarrollar en el proceso de entrenamiento la forma deportiva.

Las capacidades coordinativas se clasifican en:

- Capacidades coordinativas generales o básicas.

- Capacidades de regulación y dirección del movimiento.

- Capacidades de adaptación y cambios motrices.

En este grupo de la clasificación es la parte más importante de este trabajo. Ellas son: Orientación, equilibrio, ritmo, acoplamiento, diferenciación, reacción, anticipación.

Antes de practicar los ejercicios deportivos relacionados con la capacidad física ya sean carreras de larga distancia, abdominales, planchas, resistencia entre otros, es necesario realizar un conjunto de ejercicios, con la finalidad de que el organismo transite desde un estado de reposo relativo a un estado de actividad determinado. Cuale dicho ejercicio es el calentamiento para la actividad físico-deportivo.

El calentamiento es una de las acciones más importantes dentro del mundo de la actividad física, ya sea para la clase de educación física, el entrenamiento deportivo, la actividad terapéutica y competencias etc.

El calentamiento es una especie de ejercicios especialmente relacionados, que son relacionados a fin de preparar al organismo para determinado trabajo, permite incrementar la excitabilidad y la labilidad de los centros nerviosos, lo que facilita nuevas relaciones temporales en el proceso de la excitación y para la activación de los hábitos motores ya adquiridos en condiciones complejas de la actividad deportiva.

Con el empleo de los medios del calentamiento se logra incrementar la actividad de los fermentos, la velocidad de las reacciones bioquímicas a escala muscular y la excitabilidad y labilidad de los mismos. Es peculiar que exista un incremento de la actividad de los órganos de la respiración y del corazón, por la salida de la sangre de los dispositivos, por la redistribución de la sangre entre los órganos que están funcionando, y los que no están, así como por el incremento de la temperatura del cuerpo. 
El contenido del calentamiento estará acorde con el deporte que se entrena, el periodo de entrenamiento en que se encuentre el atleta, la finanza que persigue la clase y otros factores, entre ellos la temperatura ambiente. Es una premisa básica, que el contenido y carácter del calentamiento siempre estará de acuerdo con el contenido y carácter principal del entrenamiento.

El calentamiento se distingue en dos secciones durante la preparatoria de la clase: Calentamiento General y Calentamiento Especifico.

Calentamiento General: Es obligatorio para todos los deportes. Su objetivo consiste en elevar el nivel de la capacidad general de trabajo de los atletas y crear en ellos condiciones para pasar al calentamiento especifico. Este calentamiento está conformado por 4 contenidos bien definidos: la lubricación, el trote, los ejercicios de estiramiento, los ejercicios de flexibilidad y los ejercicios de fuerza general y puede tener una duración de 10 a 45 minutos en la clase de entrenamiento, mientras que en la de educación física estará alrededor de 3 a 6 minutos, en dependencia de los objetivos que se trace y la duración de la clase.

El complejo de ejercicios preparatorios del calentamiento se selecciona teniendo en cuenta la especialización y el nivel de preparación de los alumnos. El número de repeticiones podrá modificarse en dependencia de los objetivos del calentamiento, del nivel de preparación del educando, de las particularidades de los ejercicios, de la temperatura, la conservación del calor por la ropa y de otros factores entre ellos el tiempo disponible. El orden de los ejercicios, si es de los planos musculares superiores a los inferiores y viceversa, depende eminentemente de que debe calentarse rimero los planos musculares pequeños y luego los mayores, pues este procedimiento conduciría a que la temperatura corporal se incremente paulatinamente.

Calentamiento Especifico: Tiene como objetivo principal preparar la actividad del sistema nervioso central y el aparato neuromuscular del alumno, para la realización de aquellos ejercicios que conforman la base de la parte principal de la clase, facilita el aumento de la capacidad del trabajo de aquellos eslabones del aparato motor, que van a participar en la actividad principal. Reactivar las huellas y elevar el nivel de desarrollo delas capacidades motrices, en particular la rapidez y la unidad con los hábitos motores, en consecuencia, la parte especifica del calentamiento debe elaborarse después de haberse elaborado la parte principal de la clase, pues de ella se deriva el calentamiento especifico de la clase. Por lo general, las clases de preparación técnica no incluye calentamiento especifico en la parte preparatoria, pues los ejercicios específicos de la parte principal actúan como tal.

El calentamiento especifico, siguiendo su denominación, estará compuesto con 4-6 ejercicios de carácter especial:

- La carrera. 
- Los ejercicios especiales del calentamiento.

- Los ejercicios del deporte que se trate.

El calentamiento puede realizarse de varias formas como:

- Sobre la marcha: Con desplazamiento en el área de trabajo.

- En el lugar: Es estacionario, utilizando formaciones en círculos o en filas.

- Continuo: Esta dada por la continuidad de los ejercicios que han sido elegidos, sin pausa, utilizándose para aquellos participantes con experiencia en la actividad.

- Por separado: Este conlleva pausas marcadas entre un ejercicio y otro que permite la explicación y la demostración de los mismos.

- Activos: Es la forma más común de realización, en la que el contenido del calentamiento son ejercicios físicos.

- Pasivos: Está basado esencialmente a masajes y ejercicios físicos combinados.

El orden de realización de los ejercicios para el calentamiento es:

- Trote.

- Ejercicios de estiramiento.

- Péndulos.

- Flexiones-Extensiones.

- Torsiones.

- Círculos.

En el calentamiento se pone en práctica las actividades físicas diseñadas y aplicadas y entre ellas están:

1. Movilidad Articular (Posición Inicial). Se incluyen ejercicios para el cuello, hombros, codos, muñecas y manos, tronco, caderas, rodillas, tobillos, ejercicios especiales sin implementos, ejercicios especiales con implementos (énfasis con pelotas de látex).

2. Estimulaciones. Se incluyen ejercicios de estimulación de piernas, muslos y pantorrillas, estimulación de piernas realizando flexión.

En la estrategia implementada se incluyen diversas cargas físicas con orientación individualizadora, tanto al aire libre como en gimnasios, en las que se incluyen ejercicios 
anaeróbicos y aeróbicos, trote, carreras con intensidad baja y moderada, y diversos ejercicios con pesas.

El calentamiento presenta efectos y beneficios sobre:

El corazón: Disminuye la frecuencia cardiaca de reposo y cuando se hace un esfuerzo, aumenta la cantidad de sangre que expulsa el corazón en cada latido. De esta manera la eficiencia cardiaca es mayor gastando menos energía para trabajar. Estimula la circulación dentro del musculo cardiaco favoreciendo la alimentación del corazón.

El Sistema Circulatorio: Contribuye a la reducción de la presión arterial. Aumenta la circulación en todos los músculos. Mejora el funcionamiento venoso proviniendo la aparición de varices.

El Metabolismo: Incrementa la secreción y trabajo de diferentes hormonas que contribuyen a la mejoría de las funciones del organismo. Colabora en el mantenimiento de una vida sexual plena.

Los Aspectos Psicológicos: Disminuye el grado de agresividad, ira, ansiedad, angustia y depresión. Disminuye la sensación de fatiga. Aumenta la sensación de bienestar y disminuye el estrés mental.

El Aparato Locomotor: Aumenta la elasticidad muscular y articular. Previene la aparición de osteoporosis. Previne el deterioro muscular producido por los años. Facilita los movimientos de la vida diaria.

El mundo busca medios para enlentecer el proceso de envejecimiento, para poder hablar de la "Eterna Juventud", que se puede vincular con los ejercicios físicos, directamente con el estilo de buda. El Sistema de Salud Pública de Cuba, en coordinación con otros organismos y organizaciones del Estado, tiene como propósito contribuir a elevar el nivel de salud y la calidad de vida de las personas de la tercera edad.

\section{Conclusiones.}

- El ejercicio físico y el calentamiento no solo pueden verse como un incremento de la temperatura corporal, sino que están unificadas varias funciones, y así podemos asegurar que a través de los ejercicios físicos que se realizan en los mismos se logren pulsaciones que deben estar entre 110 y 130 minutos.

- El ejercicio físico es una actividad que nos ayuda a estar en forma y muy saludables en cuanto al corazón, al aparato locomotor, al sistema cardiovascular y a los aspectos psicológicos. 
- Las capacidades físicas nos ofrecen la posibilidad de mejorar las capacidades del cuerpo y además representa uno de los componentes esenciales para el desarrollo de las capacidades de rendimiento físico del individuo.

\section{Referencias bibliográficas.}

Gimnasia Básica. Colectivo de autores. Editorial Ciencias Médicas. La Habana 2009.

Ejercicio Terapéuticos, Fundamentos y técnicas. Quinta Edición. Carolyn Kisner.

Vía tecnológica: Infomet y Ecu Red.

López Álvarez, M et al. Efectividad del ejercicio físico en la longevidad. Policlínico "Hermanos Cruz". Pinar del Río, 2003. Rev. Ciencias Médicas, Jun 2005, vol.9, no.2, p.52-61. ISSN 1561-3194

Linares Hernández L, Mena Ramírez S, Hernández Rodríguez Y, Guerra Paredes M, Pedro Rogert F A. Relación de la cultura física terapéutica con determinadas categorías filosóficas. Rev. Ciencias Médicas [Internet]. 2007 Dic [citado 2019 Mar 11]; 11(5): 99-104.

Obando Mejía, Ibeth Aracely et al. Efecto de las actividades físicas en la disminución del estrés laboral. Rev. Cubana Med Gen Integr, Set 2017, vol.33, no.3, p.345-351. ISSN 0864-2125

«World's Most Popular Sports» (en inglés). Consultado el 31 de octubre de 2011.

Sports History of China

History of Sports in Ancient Egypt

http://www.kuwaitinfo.com/newsnew/NewsDetails1.asp?id=78319\&dt=10/13/2006\&ntype $=$ Worl

International Olympic Committee - Olympic Games

Gutiérrez, M. (1996). «¿Por qué no utilizar la actividad física como transmisor de valores sociales y personales?». Revista Española de Educación Física y Deportes 3 (1): pp. $39-42$.

Gómez, A. (2001). «Deporte y Moral: Valores Educativos del Deporte Escolar».

Ramírez Hurtado, José Manuel; Ordaz Sanz, José Antonio; Rueda Cantuche, José Manuel (Junio de 2007). «Evaluación del impacto económico y social de la celebración de grandes eventos deportivos a nivel local: el caso del Campeonato de Tenis femenino de la ITF en Sevilla en 2006» (PDF). Revista de Métodos Cuantitativos para la 
Vol. 2, N4, p. 29-46, octubre-diciembre, 2019

Economía y la Empresa: pp. 20-39. ISSN 1886-516X. http://casa.jlab.org/seminars/2009/Viewgraphs/dumas_20Apr09.pdf.

Moscoso Sánchez, David y Moyano Estrada, Eduardo (coordinadores); Biedma Velázquez, Lourdes; Fernández-Ballesteros García, Rocío;Martín Rodríguez, María; Ramos González, Carlos; Rodríguez-Morcillo Baena, Luís; Serrano del Rosal, Rafael. «Deporte, salud y calidad de vida» (PDF). Colección Estudios Sociales (Fundación $\mathrm{La}$

http://multimedia.lacaixa.es/lacaixa/ondemand/obrasocial/pdf/estudiossociales/vol2 6_sencer_es.pdf.

Caldwell, Geoffrey (1982). «Deporte internacional e identidad nacional». Revista Internacional de Ciencias Sociales (UNESCO) XXXIV (2): pp. 197-208. ISSN 03790762.

Alabarces, Pablo; Rodríguez, María Graciela (1998). Lecturas: Educación Física y Deportes (10). http://www.efdeportes.com/efd10/pamr10.htm.

Lüschen, Günter (1982). «Deporte internacional e identidad nacional». Revista Internacional de Ciencias Sociales (UNESCO) XXXIV (2): pp. 209-222. ISSN 0379-0762.

«Violencia en el deporte». Consultado el 22 de noviembre de 2011.

Jiménez Fornelas, René Alejandro. «La delincuencia juvenil: fenómeno de la sociedad actual» (PDF). Papeles de Población (Universidad Autónoma del Estado de México). ISSN 1405-7425. http://redalyc.uaemex.mx/pdf/112/11204310.pdf.

\section{【 Clencia}


PARA CITAR EL ARTÍCULO INDEXADO.

Velasteguí López, E., García Espinosa, J., Mercedes Espinosa, L., Martínez Capdevila, W. M., Hernández, Y., \& González Suarez, Y. A. (2019). El ejercicio físico como un todo para la salud humana. Anatomía Digital, 2(4), 29-46. https://doi.org/10.33262/anatomiadigital.v2i4.1122

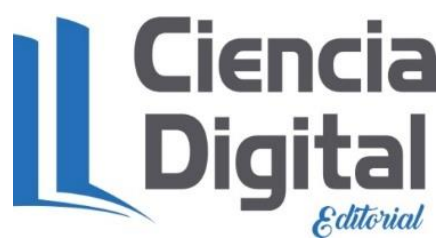

El artículo que se publica es de exclusiva responsabilidad de los autores y no necesariamente reflejan el pensamiento de la Revista Anatomía Digital.

El artículo queda en propiedad de la revista y, por tanto, su publicación parcial y/o total en otro medio tiene que ser autorizado por el director de la Revista Anatomía Digital.
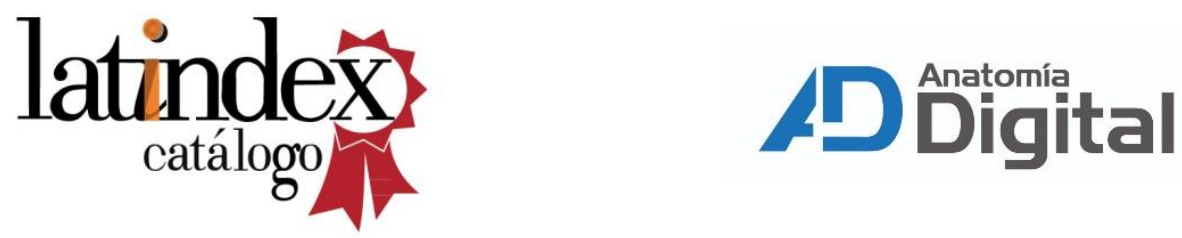